\title{
A Blue Tit Cyanistes caeruleus population: its recent increase and breeding data
}

\author{
En blåmespopulation under 60 år: dess sentida ökning samt häckningsdata
}

KARL-GUSTAV SCHÖLIN \& HANS KÄLLANDER

\begin{abstract}
Before 1983, Blue Tits Cyanistes caeruleus never made up more than four pairs of the breeding population in a nestbox study carried out in South Central Sweden. From five breeding pairs in 1983, the population increased to 29 pairs in 2007 and remained high to the end of the study in 2011. Mean laying date was strongly correlated with mean April temperature, which increased during 1983-2011. During the same period mean laying date became nearly ten days earlier. Mean clutch size was 9.90 eggs but varied both within and

between years and showed a negative relationship to population size. The mean number of fledglings varied strongly between years, partly because of predation but also due to nestling starvation.

Karl Gustav Schölin, Kyrkvärdsg. 10, SE-702 84 Örebro. Email: kalle.scholin@gmail.com

Hans Källander, Department of Biology, Lund University, Ecology Building, SE-223 62 Lund, Sweden. E-mail: hans. kallander@telia.com
\end{abstract}

Received 4 January 2012, Accepted 10 April 2012, Editor : S. Svensson

\section{Introduction}

The Swedish Blue Tit Cyanistes caeruleus population has increased by c. 30 to 50 percent during the last 30 years (Ottvall et al. 2009) and the species has also spread northwards so that it now breeds not only along the northern Baltic coast but also in inland villages north of the Arctic circle (http://www. artportalen.se). In the present study, we show how this recent population increase is reflected in data from a nestbox study in South Central Sweden spanning a little more than 60 years. We also report data on laying date, clutch size and breeding success. In two earlier papers (Schölin 2009, Schölin \& Källander 2011), corresponding data on Great Tits $P a$ rus major and Pied Flycatchers Ficedula hypoleuca from the same study plot have been presented.

\section{Study area and methods}

The study area has been described in Schölin (2009) and Schölin \& Källander (2011). In short, 80 nestboxes were put up prior to the breeding season in 1948 over a large area of mixed deciduous-conifer forest some $5 \mathrm{~km}$ to the SE of Örebro, South Central Sweden $\left(59^{\circ} 14^{\prime} \mathrm{N}, 15^{\circ} 13^{\prime} \mathrm{N}\right)$. Boxes were placed about $40-50 \mathrm{~m}$ apart at a height of at least $2 \mathrm{~m}$, many of them at forest edges and at small clearings.
The boxes had an entrance hole with a diameter of $30 \mathrm{~mm}$, a bottom area of $\mathrm{c} .110 \mathrm{~cm}^{2}$ and their base was about $16 \mathrm{~cm}$ below the lower rim of the entrance hole. Most were wooden boxes while about a third were hollowed-out tree trunks. Boxes were checked about once a week during the breeding season, sometimes somewhat less often. Clutch initiation date was back-calculated assuming the laying of one egg a day. The number of nestboxes varied during the course of the study from a maximum of 100 in 1951 to a lowest figure of 58 in 1961, but was mostly 60-70. The number of available nestboxes clearly could influence the number of pairs, but since Blue Tits are the earliest of the species after the Coal Tit Periparus ater to start breeding, and the Coal Tits were very few in each year ( $\max$ 6 pairs), below we use the uncorrected number of pairs in the analyses. All data refer to first broods. Temperature data were taken from SMHI's weather station at Örebro, some $5 \mathrm{~km}$ to the north-west.

\section{Results}

The Blue Tit population

During 1948-1982, there were never more than four pairs of Blue Tits breeding in the nestboxes in any one year. However, from 1983 (5 pairs) the 


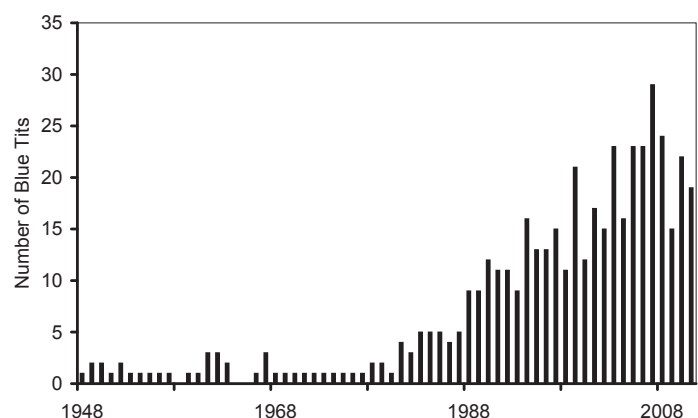

Figure 1. The number of Blue Tit pairs during 1983-2011. Antal blåmespar 1983-2011.

population increased to a top record of 29 in 2007 , after which it varied from 15 to 24 pairs during the remaining four years (Figure 1).

\section{Laying date}

Mean laying date (mean of the means) was 8.8 May for the period $1948-1982(\mathrm{~N}=32$ years, no Blue Tits in some years) vs 4.7 May during 19832011 (29 years). Because only none to four pairs bred each year during 1948-1982, here we analyse only data collected from 1983 onwards. Mean laying dates were strongly related to mean April temperature $\left(\mathrm{Y}=-1.75 \mathrm{X}+44.49, \mathrm{R}^{2}=0.49, \mathrm{df}=27\right.$, $\mathrm{P}<0.001$, Figure 2) and became earlier during the period $\left(\mathrm{Y}=-0.34 \mathrm{X}+39.85, \mathrm{R}^{2}=0.42, \mathrm{df}=27\right.$, $\mathrm{P}<0.001$, Figure 3 ). This earlier laying corresponded to an increase in mean April temperature during the same years $\left(\mathrm{Y}=0.14 \mathrm{X}+3.46, \mathrm{R}^{2}=0.45\right.$, df $=27, \mathrm{P}<0.001$, Figure 4$)$. The earliest clutch was started on 17 April $(2007,2009)$.

\section{Clutch size}

The overall mean clutch size was 9.90 eggs $(\mathrm{N}=$ 461 clutches). Mean clutch size varied from 8.55 in 1991 to 10.73 in 1998. Individual clutches varied from 3 to 15 eggs (Table 1), with clutches of 10 eggs being most frequent followed by those of nine eggs. Mean clutch size showed a negative relationship with population density $(\mathrm{Y}=-0.04 \mathrm{X}+10.28$, $\mathrm{R}^{2}=0.22$, df $27, \mathrm{P}<0.01$ ), but not with laying date or mean April temperature. Within years, clutch size decreased with the progress of the season in some but not in most years.

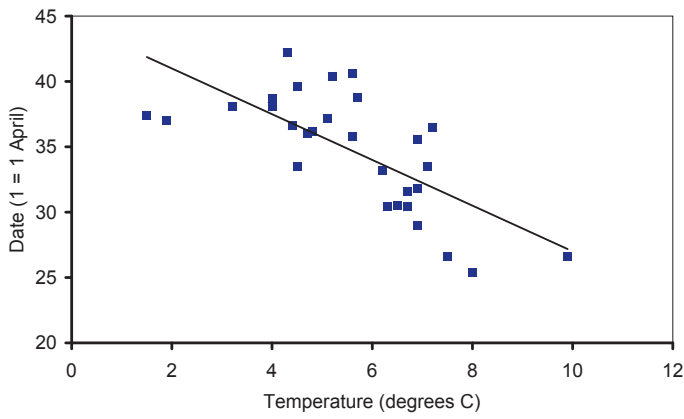

Figure 2. The relationship between Blue Tit mean laying date and mean April temperature.

Sambandet mellan medeldatum för blåmesarnas första ägg och medeltemperatur $i$ april.

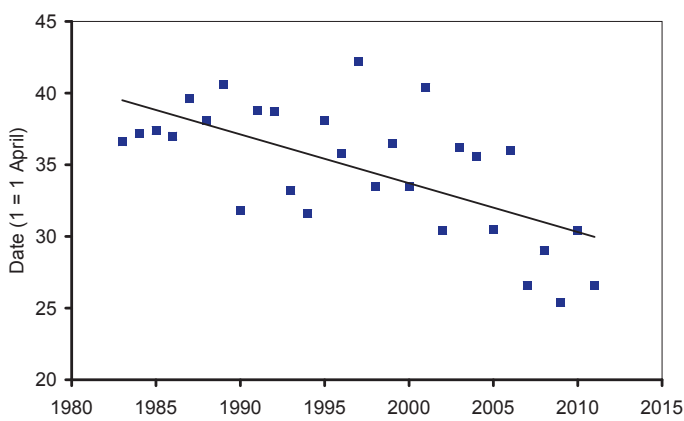

Figure 3. Mean Blue Tit laying dates during 1983-2011. Medeldatum för första ägg hos blåmesarna 1983-2011.

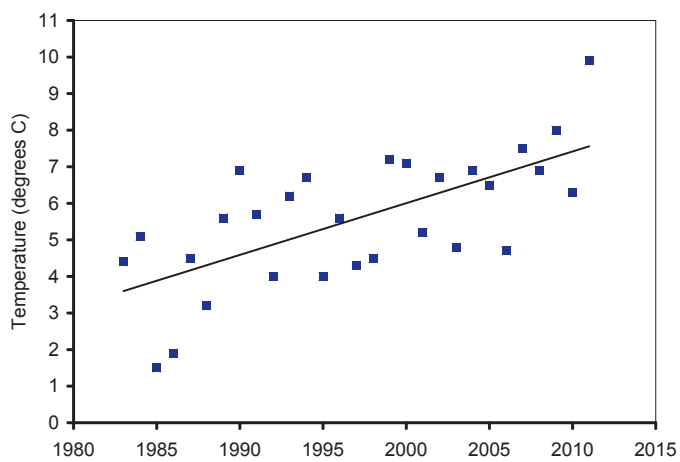

Figure 4. Mean April temperatures during 1983-2011 at Örebro, South Central Sweden.

Medeltemperatur för april åren 1983-2011. 
Breeding success

When all clutches are included, i.e. also clutches from which no young fledged, an unweighted mean for all years was 6.73 fledged young per breeding attempt, while the lowest mean number of fledglings was 4.06 in 1994. The corresponding mean for clutches that produced at least one young was 7.91 and the lowest figure was 5.00 in 1991 (Table 2). Twenty-two nests were totally depredated by Pine Marten Martes martes during 1989 to 2010, while in another nine nests 38 young out of $124(36 \%)$ survived marten attacks (Table 3 ). However, also in some years without marten predation, many broods produced few fledglings, apparently because of nestling starvation. Thus in 1991, from three clutches (22 eggs) only three young fledged, and in 2003 only 18 young fledged from five clutches (67 eggs).

\section{Discussion}

This study provides data on the increase of the Swedish Blue Tit population that has occurred during the last three decades. These data suggest that the increase may have been even stronger than that recorded in the point counts of the Swedish Bird Monitoring Programme (Ottvall et al. 2009). Also Svensson et al. (2010) recorded an increase from a mean of 3.07 pairs during $1953-1982$ to 7.85

Table 1. Number of Blue Tit clutches of different sizes during 1948-1982 and 1983-2011 together with mean clutch sizes.

Antal blåmeskullar av olika storlek samt medelkullstorlek 1948-1982 och 1983-2011.

\begin{tabular}{|c|c|c|c|c|c|c|c|c|c|c|c|c|c|c|c|}
\hline & \multicolumn{13}{|c|}{$\begin{array}{l}\text { No. of clutches with } 3,4,5 \text {, etc. eggs } \\
\text { Antal kullar med 3,4,5, etc. ägg }\end{array}$} & \multirow{2}{*}{\multicolumn{2}{|c|}{$\begin{array}{cc}\text { No. of } & \text { Mean } \\
\text { clutches } & \text { clutch size }\end{array}$}} \\
\hline & 3 & 4 & 5 & 6 & 7 & 8 & 9 & 10 & 11 & 12 & 13 & 14 & 15 & & \\
\hline $\begin{array}{l}1948- \\
1982\end{array}$ & & & & & & & 6 & 16 & 18 & 8 & 1 & & & 49 & 10.63 \\
\hline 1983 & & & & & & & 1 & 3 & 1 & & & & & 5 & 10.00 \\
\hline 1984 & & & & & & & 1 & 2 & 1 & 1 & & & & 5 & 10.40 \\
\hline 1985 & & & & & & 1 & 1 & 2 & 1 & & & & & 5 & 9.60 \\
\hline 1986 & & & & & & & 1 & 1 & 1 & 1 & & & & 4 & 10.50 \\
\hline 1987 & & & & & & & 2 & 2 & 1 & & & & & 5 & 9.80 \\
\hline 1988 & & & & & & & 1 & 5 & 3 & & & & & 9 & 10.22 \\
\hline 1989 & & & & & & 1 & 3 & 2 & 1 & 1 & 1 & & & 9 & 10.11 \\
\hline 1990 & & & & & & 1 & 4 & 5 & 2 & & & & & 12 & 9.67 \\
\hline 1991 & & & 1 & 1 & & 2 & 4 & 2 & 1 & & & & & 11 & 8.55 \\
\hline 1992 & & & & & 1 & & 4 & 3 & 2 & & 1 & & & 11 & 9.82 \\
\hline 1993 & & & & & & 2 & 1 & 2 & 3 & 1 & & & & 9 & 10.00 \\
\hline 1994 & & & & & 2 & 3 & 4 & 3 & 3 & 1 & & & & 16 & 9.31 \\
\hline 1995 & & & & & & & 3 & 5 & 3 & 2 & & & & 13 & 10.31 \\
\hline 1996 & & & & & & 2 & 3 & 1 & 4 & 2 & & & 1 & 13 & 10.46 \\
\hline 1997 & & & & & 1 & 1 & 5 & 4 & 3 & 1 & & & & 15 & 9.67 \\
\hline 1998 & & & & & & & 2 & 3 & 2 & 4 & & & & 11 & 10.73 \\
\hline 1999 & & & & & 1 & 4 & 4 & 7 & 4 & & & 1 & & 21 & 9.67 \\
\hline 2000 & & & & & & 3 & 2 & 4 & & 2 & 1 & & & 12 & 9.92 \\
\hline 2001 & & & & 1 & & 1 & 5 & 7 & 2 & 1 & & & & 17 & 9.59 \\
\hline 2002 & & & & & 1 & & 4 & 6 & 2 & 1 & 1 & & & 15 & 10.00 \\
\hline 2003 & & & & 1 & 1 & 2 & 10 & 6 & & 2 & & 1 & & 23 & 9.43 \\
\hline 2004 & & & 1 & 1 & 1 & 3 & 4 & 6 & & & & & & 16 & 8.63 \\
\hline 2005 & & & & & 4 & 3 & 6 & 7 & 1 & 2 & & & & 23 & 9.17 \\
\hline 2006 & & & 1 & 1 & 4 & 2 & 5 & 7 & 1 & 2 & & & & 23 & 8.91 \\
\hline 2007 & & & & & 1 & 2 & 9 & 12 & 4 & 1 & & & & 29 & 9.66 \\
\hline 2008 & & & & & 2 & 5 & 5 & 8 & 4 & & & & & 24 & 9.29 \\
\hline 2009 & & & & & 1 & 2 & 2 & 3 & 5 & 2 & & & & 15 & 10.00 \\
\hline 2010 & & & & & 2 & 2 & 4 & 5 & 6 & 3 & & & & 22 & 9.91 \\
\hline 2011 & 1 & & 1 & & 1 & 2 & 2 & 8 & 2 & & 2 & & & 19 & 9.32 \\
\hline Total & 1 & & 4 & 5 & 23 & 44 & 108 & 160 & 79 & 36 & 7 & 2 & 1 & 461 & 9.90 \\
\hline
\end{tabular}


Table 2. Number of Blue Tit broods from which zero, one, two, three, etc. young fledged and mean number of fledglings from all broods (1) and from broods where at least one young fledged (2). Total number of broods 470. Antal blåmeskullar från vilka ingen, en, två, tre, osv. ungar blev flygga samt medeltalet flygga ungar från alla kullar (1) och från kullar där minst en unge blev flygg (2). Totalt antal kullar 470.

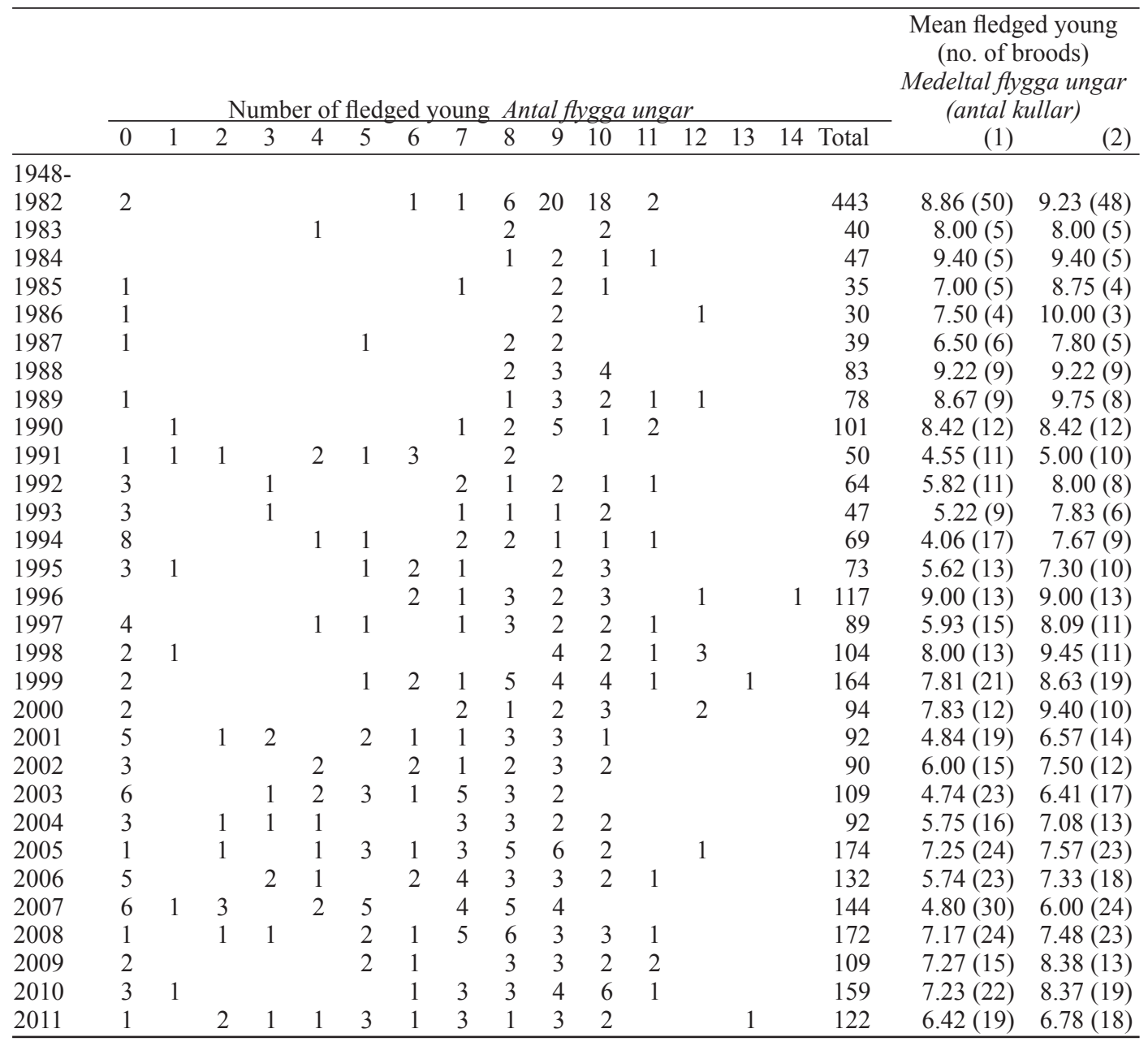

pairs during 1983-2009 in Fågelsångsdalen, a 13 ha wooded stream valley near Lund, South Sweden. The increase during the last three decades is most likely an effect of a warmer climate, especially during the winter months. Also the Blue Tit's northward expansion of its breeding range during the same period supports this hypothesis even though a more widespread winter feeding cannot be completely ruled out (Ottvall et al. 2009). Both in the Netherlands and in Finland Blue Tit populations have shown an upward trend since the 1960s. This trend is strong in the material from the Finnish winter bird census (Hildén 1990). In the Nether- lands, the trend was present in two mixed forests, where Blue Tit density was low, but not in two high density oak woods (van Balen \& Potting 1990). In the present study, however, Blue Tits were few until the early 1980 s.

Mean laying date was about four days later in 1948-1982 than in 1983-2011, but became successively earlier by almost 10 days from 1983 to the end of the study. This earlier laying coincided with a positive trend in April temperatures in agreement with a close association between laying date and ambient temperature.

The mean clutch size (9.90 eggs) was somewhat 
lower than in a study in southern Finland (11.23 eggs, with a span from 10.71 to 11.81 eggs; Hildén $1990)$ and in Estland (11.3; listed in Glutz \& Bauer 1993), both studies at comparable latitudes to Örebro. Clutch size in Blue Tits seems to be influenced by breeding habitat, as discussed in Glutz \& Bauer (1993), who also present data on clutch sizes from various parts of Europe and North Africa.

Productivity, i.e. mean number of fledglings, was low in many years. This was partly the result of predation by martens but was also caused by nestling starvation and desertions. The latter two factors may indicate that the habitat was sub-optimal for Blue Tits, with a relatively high proportion of conifers and with the broad-leaved trees and shrubs dominated by birch Betula spp., aspen Populus tremula, alder Alnus glutinosa and rowan Sorbus aucuparia. Oak Quercus robur, which seems to be the most important tree for Blue Tits in the British Isles (Perrins 1979) and in continental Europe (Glutz \& Bauer 1993), was absent in the area.

\section{References}

Balen, J.H. \& Potting, R.P.J. 1990. Comparative reproductive biology of four Blue Tit populations in the Netherlands. Pp. 19-38 in Population Biology of Passerine Birds (Blondel, J., Gosler, A., Lebreton, J.-D. \& McCleery, R., eds). NATO ASI Series, Series G: Ecological Sciences, Vol. 24.

Glutz von Blotzheim, U.N. \& Bauer, K.M. 1993. Handbuch der Vögel Mitteleuropas. Bd 13/I. AULA-Verlag, Wiesbaden.

Hildén, O. 1990. Long-term study of a northern population of the Blue Tit Parus caeruleus. Pp. 65-75 in Population Biology of Passerine Birds (Blondel, J., Gosler, A., Lebreton, J.-D. \& McCleery, R., eds). NATO ASI Series, Series G: Ecological Sciences, Vol. 24.

Ottvall, R., Edenius, L., Elmberg, J., Engström, H., Green, M., Holmqvist, N., Lindström, Å., Pärt, T. \& Tjernberg, M. 2009. Population trends for Swedish breeding birds. Ornis Svecica 19: 117-192.

Perrins, C.M. 1997. British Tits. Collins, London.

Schölin, K.G. 2009. A long-term study of the breeding biology of the Great Tit Parus major in a mixed forest in South Central Sweden. Ornis Svecica 19: 222-232.

Schölin, K.G. \& Källander, H. 2011. A 64-year study of a Pied Flycatcher Ficedula hypoleuca population. Ornis Svecica 21: 79-91.

Svensson, S., Thorner, A.M. \& Nyholm, N.E.I. 2010. Species trends, turnover and composition of a woodland bird community in Southern Sweden during a period of fiftyseven years. Ornis Svecica 20: 31-44.

\section{Sammanfattning}

Den svenska blåmespopulationen har ökat med 30-50 procent under de senaste tre decennierna
Table 3. Pine Marten predation of Blue Tit broods at Lövsätter during 1989 to 2010 . The right column presents partial predation.

Mårdpredation på blåmeskullar vid Lövsätter 1989 2010. Den högra kolumnen redovisar partiellt prederade kullar.

\begin{tabular}{ccc}
\hline Year & $\begin{array}{c}\text { No. of totally } \\
\text { depredated nests } \\
\text { Totalförluster } \\
\text { av ungkullar }\end{array}$ & $\begin{array}{c}\text { No. of young taken } \\
\text { (original brood size) } \\
\text { Antal tagna ungar } \\
\text { (ursprungligt antal) }\end{array}$ \\
\hline 1989 & 1 & $8(11)$ \\
1993 & 3 & $6(10)$ \\
1994 & 5 & $5,4,6(10,10,9)$ \\
1995 & 3 & $5,7(9,12)$ \\
1997 & 3 & \\
1998 & 1 & $5(8)$ \\
2000 & 1 & $2(7)$ \\
2006 & 1 & \\
2007 & 1 & 48 \\
2008 & 1 & \\
2010 & 1 & \\
\hline Total & 21 & \\
\hline
\end{tabular}

(Ottvall m.fl 2009) och arten har också spritt sig norrut så att den numera häckar inte bara längs Östersjökusten utan också i byar i inlandet norr om polcirkeln (http:/www.artportalen.se/birds). I följande sammanställning visar vi hur denna sentida populationsökning speglas i data från en holkstudie i Mellansverige som spänner över mer än 60 år. Vi rapporterar också data om läggdatum, kullstorlek och häckningsframgång. I två tidigare uppsatser (Schölin 2009, Schölin \& Källander 2011) har vi presenterat motsvarande data för talgoxe Parus major och svartvit flugsnappare Ficedula hypoleuca.

\section{Studieområde och metoder}

Studieområdet har beskrivits i Schölin (2009) och Schölin \& Källander (2011). I korthet sattes 80 holkar upp inför häckningssäsongen 1948 i en blandskog av barr- och lövträd cirka $5 \mathrm{~km} \mathrm{SO}$ om Örebro. Holkarna placerades med 40-50 m mellanrum på minst $2 \mathrm{~m}$ höjd i träden, många av dem i skogsbryn och gläntor i skogen. Holkarna hade ett ingångshål om $30 \mathrm{~mm}$ diameter, en bottenyta av cirka $110 \mathrm{~cm}^{2}$ och holkbotten var ungefär $16 \mathrm{~cm}$ under ingångshålets nedre kant. De flesta holkarna var vanliga brädholkar, men cirka en tredjedel utgjordes av urholkade stammar. Holkarna kontrollerades ungefär en gång i veckan under häckningssäsongen, ibland något mindre ofta. Datum för 
första äggets läggande bestämdes genom att räkna bakåt från iakttaget äggantal under antagande att ett ägg lagts per dag. Antalet holkar varierade under studiens gång från ett maximum av 1001951 till det lägsta antalet, 58, 1961, men var vanligen 60-70. Antalet tillgängliga holkar kan givetvis påverka antalet par, men eftersom blåmesen är den art efter svartmesen Periparus ater, som påbörjar häckningen tidigast, och svartmesarna var mycket få varje år (max 6 par), har vi i analyserna nedan använt okorrigerade siffror för antalet par. Temperaturdata har tagits från SMHI:s väderstation i Örebro, ungefär 5 km nordväst om studieområdet.

\section{Blåmespopulationen}

Under åren 1948-1982 häckade inte under något år mer än fyra par blåmesar i holkarna. Från och med 1983 (5 par) ökade emellertid populationen till rekordantalet 29 par 2007. Därefter varierade antalet från 15 till 24 par under de följande fyra åren (Figur 1).

\section{Läggdatum}

Medeldatum för första ägget läggande 1948-1982 (32 år) var den 8,8 maj mot 4,7 maj 1983-2011 (29 år). Eftersom bara noll till fyra par häckade varje år under perioden 1948-1982, så analyserar vi här endast data från och med 1983. Medeldatum för första ägget var starkt relaterat till medeltemperaturen under april och lades 1,75 dagar tidigare för varje grads temperaturökning (Figur 2). Äggläggningsstarten blev allt tidigare under perioden (c.10 dagar, Figur 3) i takt med en ökning av den genomsnittliga apriltemperaturen (Figur 4). Den tidigaste kullen startades den 17 april $(2007,2009)$.

\section{Kullstorlek}

Kullstorleken, baserad på alla kullar, var i medeltal 9.90 ägg $(\mathrm{N}=461$ kullar $)$ och varierade från 3 till 15 ägg (Tabell 1), med 10-äggskullar som de vanligast förekommande, följda av kullar med nio ägg. Medelkullstorleken minskade något (med 0.04 ägg) med ökande antal par men inte med medelläggdatum eller genomsnittlig apriltemperatur. Under vissa år minskade kullstorleken allt eftersom säsongen framskred, men oftast inte.

\section{Häckningsframgång}

När alla kullar inkluderas, dvs också kullar från vilka inga ungar blev flygga, var det ovägda medel- antalet flygga ungar 6,73. Det lägsta antalet flygga ungar noterades 1994 med i medeltal endast 4,06 flygga. Motsvarande för kullar där minst en unge blev flygg, var 7,71 och den lägsta siffran $(5,00)$ noterades 1991 (Tabell 2). Mellan 1989 och 2010 tog mård samtliga ungar i 22 bon, medan i ytterligare nio bon 38 ungar av $124(36 \%)$ överlevde mårdpredationen (Tabell 3). Men också vissa år utan mårdpredation producerade många kullar få ungar, uppenbarligen på grund av svält. Sålunda gav 199122 ägg (tre kullar) endast upphov till tre flygga ungar, och 2003 blev bara 18 ungar flygga från 67 ägg (fem kullar).

\section{Diskussion}

Denna studie presenterar data som illustrerar den ökning av den svenska blåmespopulationen som skett under de senaste tre decennierna. Data antyder att ökningen kan ha varit ännu starkare än vad som dokumenterats med hjälp av punktrutterna i det svenska fågelövervakningsprogrammet (Ottvall m.fl. 2009). Också Svensson m.fl. (2010) registrerade en ökning från i medeltal 3,07 par 1953-1982 till 7,85 par 1983-2009 i Fågelsångsdalen, en 13 ha stor skogklädd bäckdal nära Lund. Ökningen under de senaste tre decennierna är högst sannolikt en effekt av ett varmare klimat, speciellt under vintermånaderna. Också expansionen av blåmesens häckningsområde norrut under samma period stöder denna hypotes även om en alltmer utbredd vintermatning inte helt kan uteslutas (Ottval m.fl. 2009).

Kullarnas första ägg lades under perioden 19481982 ungefär fyra dagar senare än under 19832011. Den senare perioden kännetecknas dock av en klar trend mot tidigare läggning, med en nästan 10 dagar tidigare läggstart i slutet än i början av perioden. Denna tidigare läggstart sammanföll med en signifikant trend mot högre apriltemperaturer.

Medelantalet flygga ungar var relativt lågt många år. Detta var delvis ett resultat av mårdpredation men orsakades också av att ungar svalt, samt av övergivningar (mest av nykläckta ungar). De senare två faktorerna indikerar möjligen att biotopen var suboptimal för blåmesen med en hög andel barrträd och med lövskog dominerad av träd och buskar som björk, asp och rönn men med avsaknad av ek, ett trädslag som anses vara optimalt för blåmesen. 